EPiC Series in Engineering
Volume 3, 2018, Pages 739-745
HIC 2018. 13th International
Conference on Hydroinformatics

\title{
Considering water footprint in reservoir adaptation to climate change: an evolutionary approach
}

\author{
Carlos O. Galvão ${ }^{1,2}$, Erica C. M. Machado ${ }^{1}$, Elpida Kolokytha ${ }^{3}$ and Haris \\ Skoulikaris ${ }^{3}$ \\ ${ }^{1}$ Federal University of Campina Grande, Campina Grande, Brazil \\ ${ }^{2}$ Griffith University, Nathan, QLD, Australia \\ ${ }^{3}$ Aristotle University of Thessaloniki, Thessaloniki, Greece \\ carlos.galvao@ufcg.edu.br, ericacristine@gmail.com, \\ lpcolecivil.auth.gr, hskoulikecivil.auth.gr
}

\begin{abstract}
Reservoir management usually considers short- to medium-term operation horizons. However, climate change and other longer term societal changes pose a challenge for planning water utilization from reservoirs. The key aspect is how to incentive behaviour change towards gradual adaptation. We propose an evolutionary approach to model adaptation, considering the Water Footprint as the main criterion for driving adaptation in long-term. The approach is tested in a case in Brazil, revealing promising preliminary results.
\end{abstract}

\section{Introduction}

Reservoir operations are usually planned for short- to medium-term horizons. Long-term adaptation requires gradual changes in water usage, and therefore allocation of releases must include criteria considering the various scale-horizons. The short-term criteria are designed to maintain economic sustainability of water-based production systems, whilst the medium- to long-term criteria are designed to foster the change in water uses towards more efficient production systems, compatible to the expected climate change. The incentives to new production systems should indicate the direction towards efficiency.

The Water Footprint (WF) approach is an interesting candidate to be the criterion regarding adaptation, within the water allocation model. The WF is a contemporary indicator to investigate water consumption and pollution along supply chains, evaluate the sustainability of water use and explore the possibility to reduce water uses or to replace pollution intensive methods (Hoekstra and Chapagain, 
2008; Hoekstra, 2017). A water footprint generally is studied by its three components: the blue, green and grey water footprint. The blue water footprint is the volume of freshwater that is evaporated from the surface and groundwater resources. The green water footprint is the volume of water evaporated from the rainwater stored in the soil. The grey water footprint is the volume of polluted water, which is quantified as the volume of water that is required to dilute pollutants to such an extent that the quality of the ambient water satisfy certain agreed water quality standards (Hoekstra and Chapagain, 2008). The high value of grey water footprint reveals the significant water quality deterioration (pollution) whereas the high value of green and blue WF indicates unsustainable water consumption.

Since the appropriation of such concepts in allocation models requires proper consideration of the objectives on a variety of time scales, an evolutionary approach seems to be appropriate. Therefore, in the present work, the WF could be introduced in the allocation model as a comprehensive indicator for adaptive water management. More specifically the WF could determine the most efficient allocation of water in the reservoir and could also determine the limits of water pollution. The WF criteria were already applied to a case of a lake in Greece (Kolokytha, 2014) and could provide defensible guidelines for mitigation of lake water overexploitation. The WF concept and the evolutionary algorithm for water allocation are used together in this paper.

\section{Water Footprint concept in water allocation}

The temporal and spatial uneven distribution of water is one of the major problems of water resources management. The problem becomes even worse due to climate change, responsible for further shortages of freshwater and increased pollution of water bodies, both acting as limiting factors in the economic and social development of many countries.

Planning water allocation requires the consideration of the environmental flow, and at the same time the water for direct human use (e.g. agriculture, power generation, domestic purposes and industry) and indirect human use (maintenance of ecosystem services) (Acreman, 1998) A comprehensive approach towards freshwater use and scarcity, should consider both green and blue water consumption (Falkenmark, 2000) as well as water pollution (Postel et al., 1996).

The Water Footprint was first introduced by Hoestra in order to evaluate how the human activities related to the utilization of water can become more sustainable. Serving the aim of raising awareness and improving sustainable water management, the WF is proved as a very useful indicator (Kolokytha, 2014). It refers to all forms of freshwater use (consumption and pollution) that contribute to the production of goods and services consumed by the users of a certain geographical region or a river basin (Hoekstra and Chapagain, 2008). The three types of water namely blue, green and grey water are analysed separately in order to evaluate water quantity (rain, ground and surface water) and water quality (grey water). The analysis of the WF in a river basin provides valuable information to facilitate the efficient allocation of water resources in different economic and environmental requirements as it provides new data in order to tackle with water scarcity and water pollution problems.

Applying the Water Footprint concept facilitates the comparison of the WFs of the water consuming and polluting production sectors (irrigation, municipal water, industry) in a specific river basin with the water availability at the same basin to improve water management decision making. The innovation of the WF concept is its grey component, providing valuable information when considering the relevance of pollution as a driver of water scarcity.

A high WF implies high water consumption affecting the capacity of the basin to fulfil all water demands. It also affects the downstream water users negatively and should be avoided, as well as it is undesirable in regions experiencing water scarcity problems. Hence, the aim is the reduction of WFs and the increase of the sustainability of WFs, i.e. environmental, social, economic and even political objectives' integration. 
By incorporating the WF into the modelling of the different flows one can study the limits/ per water use in order to not exceed the availability of existing renewable water resources or to foster climate change impact on water resources at river basin scale and at the same time identify sources of pollution.

\section{Materials and Methods}

\subsection{Evolutionary allocation model}

This paper proposes a multi-objective model, adapted from a previous approach (Machado et al., 2012), for allocation of basin flows, which considers different uses, quantity and quality of water as well. It takes account of not only releases and abstractions from the reservoir but also all water uses within the river basin, both upstream and downstream of the reservoir. This model was developed with the purpose of being easily coupled with others that represent the hydro-meteorological system of the catchment area. In addition to promoting the use of more efficient water availability, through the expansion of the volumes granted, it can also be used in defining the rationing volume for each user in times of scarcity.

Its main feature, which differentiate it from other algorithms, is that it handles the problem constraints throughout the evolution process, from generation of the initial population, performance assessment and the crossover and mutation operators. This approach innovates in considering the concepts of dominance and viability of candidate solutions within the selection and crossover operators, whilst still preserving traditional operators to guarantee diversity in the candidate solutions.

The model uses two objective functions, as described below:

1) Objective Function 1: This function aims at maximizing the users' satisfaction, approximating the allocated volume to the desired by the users. Introduces the WF as a coefficient (CWF). The lower is the user's WF, the higher is its CWF. The rationale behind this formulation is to give additional allocation as a bonus to those who have a lower WF (1).

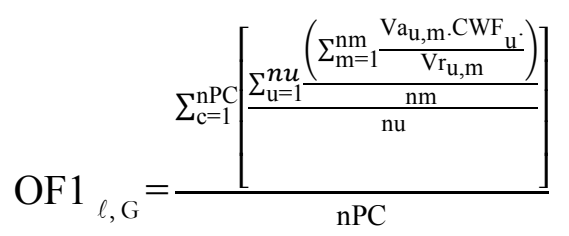

FO1 $1_{\ell, \mathrm{G}}$ is the objective function of the users' satisfaction, of the individual $\ell$ in the interval of time $\mathrm{G}$; $\mathrm{nPC}$ is the number of region in the basin; $\mathrm{nu}$ is the number of users in the region; $\mathrm{Va}_{\mathrm{u}, \mathrm{m}}$ is the water allocated for the user $u$ in the interval of time $m\left[\mathrm{~m}^{3}\right]$; $\mathrm{Vr}_{\mathrm{u}, \mathrm{m}}$ is the water required by user $u$ in the interval of time $m\left[\mathrm{~m}^{3}\right]$;

$\mathrm{Nm}$ is the number of interval of time; $\mathrm{CWF}_{\mathrm{u}}$ is the WF coefficient of user $u$;

2) Objective Function 2: The second objective function minimises the pollutant emissions, through the maximisation of the resulting concentration between the effluent load and river/reservoir water, considering the maximum allowed concentration by the environmental agency (2). 
$\mathrm{OF} 2_{\ell, \mathrm{G}}=\left\{\begin{array}{c}\text { if, } \mathrm{Cr}_{\mathrm{p}, \mathrm{m}}>\mathrm{Ca}_{\mathrm{p}, \mathrm{m}}: \frac{\sum_{\mathrm{p}=1}^{\mathrm{nP}}\left[\frac{\left(\sum_{\mathrm{m}=1}^{\mathrm{nm}} \frac{\mathrm{Ca}_{\mathrm{p}, \mathrm{m}}}{\mathrm{Cr}, \mathrm{m}}\right)}{\mathrm{nm}}\right]}{\mathrm{nP}} \\ \text { else: } \frac{\sum_{\mathrm{p}=1}^{\mathrm{nP}}\left[\frac{\left(\sum_{\mathrm{m}=1}^{\mathrm{nm}} \frac{\left.\mathrm{Cr}_{\mathrm{p}, \mathrm{m}}+1\right)}{\mathrm{Ca}, \mathrm{m}}\right)}{\mathrm{nm}}\right]}{\mathrm{nP}}\end{array}\right.$

FO2 $2_{\ell, G}$ is the objective function of minimises the pollutant emissions, of the individual $\ell$ in the interval of time $\mathrm{G}$;

$\mathrm{nP}$ is the number of region with contribution of biochemical oxygen demand - BOD;

$\mathrm{Cr}_{\mathrm{p}, \mathrm{m}}$ is the BOD concentration after BOD emission, [mg/l];

$\mathrm{Ca}_{\mathrm{p}, \mathrm{m}}$ is the BOD concentration maximum permissible $[\mathrm{mg} / \mathrm{l}]$.

Since the Evolutionary Model is a stochastic optimization method, its operators use random variables in their processing. Thus, usually three runs are performed for each condition/scenario, the average taken as the result.

\subsection{Study area}

The allocation model is applied at the Gramame River basin, with $600 \mathrm{~km}^{2}$, located in the coastal region of the State of Paraíba, in Brazil. The basin was divided into ten regions (Figure 1). For each region, an upper limit of water abstraction is established in the basin master plan. Municipal water supply constitutes the most significant use of water, around $58 \%$, followed by irrigation, with $41 \%$ of the total demand.

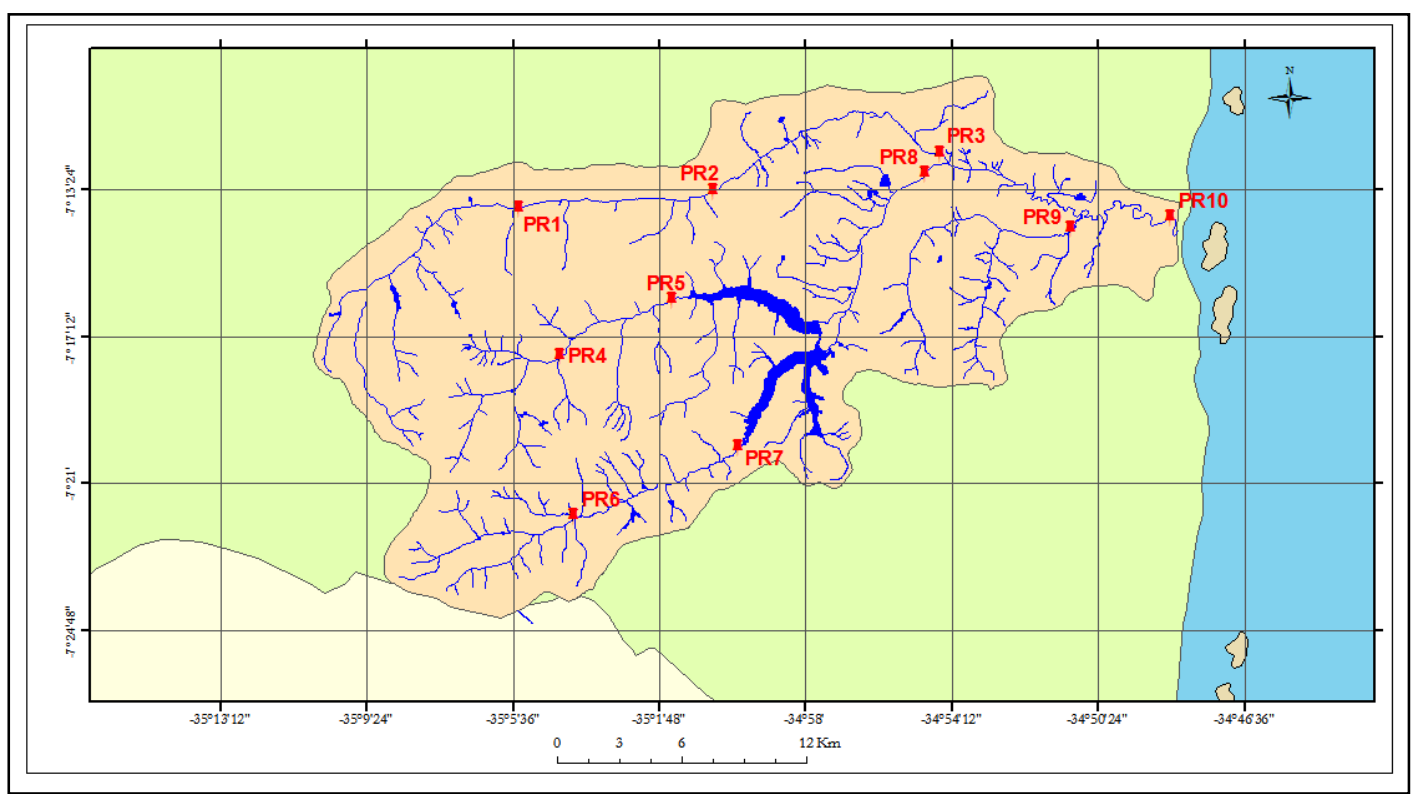

Figure 1: Gramame river basin, regions' reference points (in red). 
The participants in the allocation process are 28 users, located over the ten reference regions (Figure 1). Among them, 22 are agricultural users of irrigation, with the request of an additional flow for the months of September to March; 2 are industrial users of abstraction, with the request of additional supply of flow for all the months of the years; 2 are industrial users of effluents, with request of contribution of dilution of their effluents for all months of the year; and 2 are municipal water supply withdrawals, with regular abstractions throughout the year.

A hypothetic situation was simulated, when the demands required cannot be met. This can be an actual case in a future under climate change. The algorithm is expected to help in deciding on guidelines towards a fair allocation among users.

The evolutionary algorithm was executed for two contrasting scenarios: (1) not considering WF $(\mathrm{CWF}=1$ for all users); and (2) considering $\mathrm{WF}(\mathrm{CWF}=1$ for agricultural users - high WF; $C W F=3$ for other users - low WF). The optimization was evaluated within a 12 months operational horizon. The values of CWF, assigned here at regular intervals, are arbitrary and illustrative. However, they should be related to the actual WF differences among users.

\section{Results and discussion}

The results for the two contrasting scenarios can be analyzed as in Table 1 and summarized in Figure 2. The allocation under the standard criteria represented by the two objective functions shows that the drastic reduction in the reservoir levels would most penalize the effluent dilution and then the municipal/industrial and, finally, the agricultural sector. The introduction of the WF criteria promotes only a slightly reduction in allocation for agriculture (about $1 \%$ ), which could be distributed to increase the allocation to the municipal/industrial sector in $5 \%$ and in $46 \%$ for effluent dilution.

Table 1: Optimization results in scenarios

\begin{tabular}{|c|c|c|c|c|c|c|}
\hline Scenario & Execution & OF1 & OF2 & $\begin{array}{l}\text { Satisfaction of } \\
\text { irrigation users }\end{array}$ & $\begin{array}{l}\text { Satisfaction of } \\
\text { effluent dilution } \\
\text { users }\end{array}$ & $\begin{array}{l}\text { Satisfaction of } \\
\text { municipal and } \\
\text { industrial users }\end{array}$ \\
\hline \multirow[t]{3}{*}{1} & 1 & 0.8172 & 1.5541 & 77.29 & 3.94 & 66.14 \\
\hline & 2 & 0.8034 & 1.4977 & 72.78 & 4.86 & 67.50 \\
\hline & 3 & 0.8088 & 1.7598 & 76.41 & 5.77 & 66.13 \\
\hline \multicolumn{2}{|c|}{ AVERAGE } & 0.8098 & 1.6039 & 75.50 & 4.86 & 66.59 \\
\hline \multirow[t]{3}{*}{2} & 1 & 1.2731 & 1.8498 & 73.63 & 2.81 & 70.57 \\
\hline & 2 & 1.2943 & 1.5662 & 75.95 & 7.64 & 69.67 \\
\hline & 3 & 1.2894 & 1.0332 & 74.50 & 10.89 & 70.01 \\
\hline \multicolumn{2}{|c|}{ AVERAGE } & 1.2856 & 1.4831 & 74.69 & 7.11 & 70.08 \\
\hline
\end{tabular}

The reduction of agricultural WFs and their impacts can be achieved by improving water productivities, changing cropping patterns and studying and modifying actual trade patterns. Moreover, crop efficiency can be improved by improving water irrigation schemes and limit water leakages. We should view the production system as an integrated multiple-use system incorporating all uses and their interrelationships.

The classification of a river basin as moderate, or significant or severe water-scarce in terms of natural runoff can be attributed in terms of percentages. This means that if the blue water footprint is higher than $20 \%$ then the river basin is classified as significant. If the blue water footprint is lower than 
$20 \%$ of the natural runoff and does not exceed the blue water availability, then the blue water scarcity is classified as moderate.

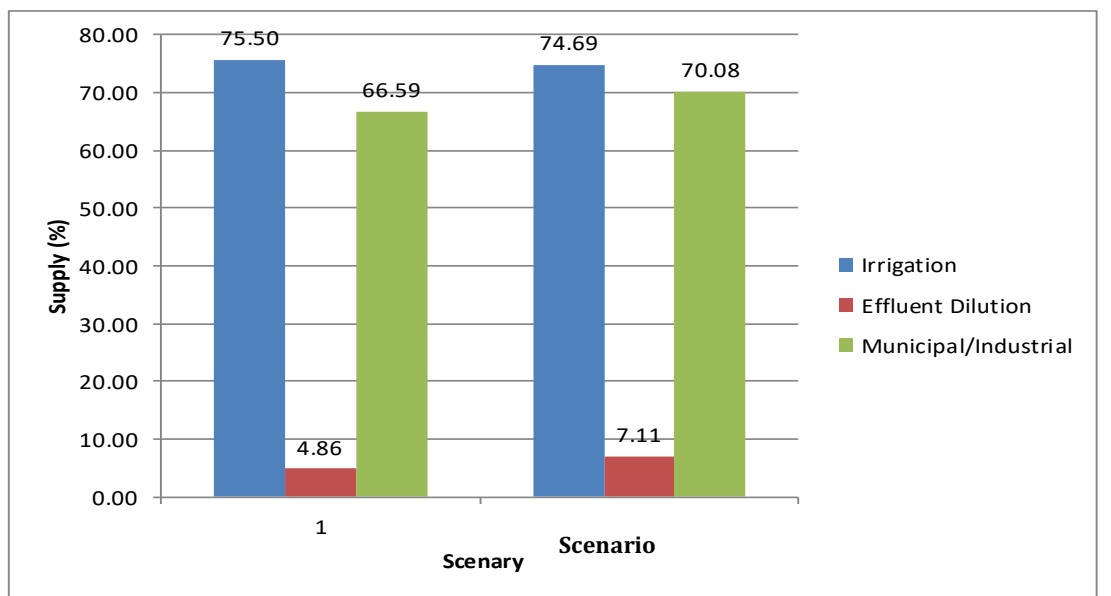

Figure 2: Water allocation, in \% of requested demands, for irrigation (in blue), effluent dilution (in red) and municipal/industrial supply (in green). Scenario 1 is the status quo and Scenario 2 introduces the WF.

In this exercise, the tentative values for CWF (1 and 3) are preliminary and were assigned to all users in each sector and, therefore, do not consider intra-sector differences in users' WF. Moreover, to express feasible and realistic adaptation guidelines, values of CWF and other variables in the objective functions must be subject to sensitivity analysis.

Hoekstra defines three levels of spatio-temporal explication in water footprint accounting (Hoekstra and Chapagain, 2008): (i) the global level, using an annual resolution, (ii) the national or regional level, using annual or monthly resolutions, and (iii) the local level, using monthly or daily resolutions. The current exercise considered monthly allocation in only one-year operational horizon. Further model improvements must consider these ranges of spatiotemporal scales to better simulate an adaptation trajectory in the long-term.

Some other critical questions that can be derived from this exercise are:

- Does the water footprint deprive other local water users (equitable allocation)?

- Is water scarcity taking into consideration in the decision to consume water (resource efficiency)?

\section{Conclusions}

An evolutionary algorithm was designed to include Water Footprint as a criterion for water allocation among different users: agricultural, municipal, industrial and effluent dilution in a basin controlled by a reservoir. The model's parameterization shows sensitivity to the new criterion, slightly reducing allocation to the agricultural users, who presented higher WF, and increasing allocated volumes to the users who have a lower WF. These results are still preliminary and, although quite evident, they point to the potential new area that is opened by introducing this innovative concept of WF in water allocation schemes. This way the reservoir management response strategy to global change in terms of resource efficiency and equitable allocation can be improved.

The sensitivity of the coefficient CWF defines the "equity" of allocation of water. A further detailed parametric analysis will reveal which is the value of CWF that could provide the sustainable water management of all respective uses. 


\section{Acknowledgements}

Authors acknowledge the Brazilian agencies CAPES, ANA, CNPq, FINEP and CT-HIDRO and the support from projects BRAMAR, INCT-MG, Cheias e Secas.

\section{References}

Acreman, M.C. (1998). Principles of water management for people and the environment. In Water and Population Dynamics, eds. A. de Shirbinin and V. Dompka. American Association for the Advancement of Science. pp: 321.

Falkenmark, M. (2000). Competing freshwater and ecological services in the river basin perspective: an expanded conceptual framework. Water Int 25(2):172-177.

Hoekstra, A. Y., Chapagain, A. K. (2008). Globalization of Water: Sharing the Planet's Freshwater Resources, Blackwell Publishing, Oxford.

Hoeskstra, A.Y. (2017). Water Footprint assessment: evolvement of a new research field, Water Resour Manage, 31, 3061-3081.

Kolokytha, E. (2014). Agricultural development in Lake Koronia. The role of the water footprint of major crops in combating climate change, Proceedings $3^{\text {rd }}$ IAHR Europe Congress, Porto.

Machado, E.C.M., Galvão, C.O., Souza Filho, F.A. (2012). Qualitative and quantitative water allocation in river basins: multiobjective methodology as part of the context of water resources management, Brazilian Journal of Water Resources, 17, 213-227.

Postel, SL, Daily, GC, Ehrlich PR (1996) Human appropriation of renewable fresh water. Science 271:785-788. 